\title{
Image-Based Visual Servoing Control for Spacecraft Formation Flying
}

\author{
Leonard Felicetti \\ Cranfield University \\ Cranfield, MK43 0AL \\ United Kingdom \\ Leonard.Felicetti@cranfield.ac.uk
}

\author{
Jorge Pomares \\ University of Alicante \\ 03690 Sant Vicent del Raspeig \\ Spain \\ jpomares@ua.es
}

\begin{abstract}
This paper proposes an image-based visual-servoing algorithm that allows for optimal formation control. The proposed distributed controller utilizes visual features of other team members, retrieved from images captured by onboard cameras, to autonomously plan and perform formation acquisition, keeping or reconfiguration maneuvers. The problems of minimization of the control effort is analyzed and the paper proposes an optimal framework for developing controllers that address the issue. The viability of such a technique is explored through numerical simulations.
\end{abstract}

\section{TABLE OF CONTENTS}

1. INTRODUCTION............................................................. 1 2. SPACECRAFT FORMATION FLYING SCENARIO..... 2 3. CAMERA MOdEL ................................................... 3 4. Optimal Visual Servoing Controller ......... 3 5. NUMERICAL SIMULATIONS.................................... 4 A. Formation keeping maneuver ................ 5 B. Formation reconfiguration maneuver ..... 6 6. CONCLUSION........................................................ 7

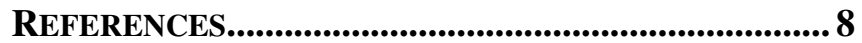
BIOGRAPHY ...............................................................99

\section{INTRODUCTION}

An increasing number of space missions nowadays require a synergic cooperation among multiple spacecraft. In some cases, payload and mission requirements impose constraints on relative distances and configurations, so that the spacecraft have to orbit in close formation. The usefulness of this architecture often lies in the spacecraft capability of maintaining defined orbital configurations for short or longer periods within a certain accuracy. In these cases, relative navigation plays a key role in the overall system performance as the knowledge of the relative kinematic states, i.e., relative positions and attitudes, of each formation member is a fundamental prerequisite for planning formation acquisition, formation reconfiguration, formation keeping or collision avoidance maneuvers [1].

Although radio frequency and GNSS receivers are commonly employed in missions implementing formation flying [2], the utilization of on-board cameras remains an appealing choice because considered as a low cost and mainly passive solution, while providing accurate and somehow independent kind source of measurements of the line-of-sight
[3] and, in some cases, of the entire relative pose of the observed spacecraft [4]. Further, the technology readiness of space-qualified cameras, as well as of the on-board computers for mid-sized satellites, is mature enough to allow for an on-board implementation of algorithms for the realtime estimation of relative pose among spacecraft [5][6]. Following the classical GNC approach, these estimations are usually used for driving the controllers to perform the required formation maneuvers. On the other hand, the still limited computing capabilities of on-board computers for small- and nano-satellites, force to employ and to develop simplified navigation algorithms for the same purposes [7].

An Image-Based Visual Servoing (IBVS) strategy is proposed in this paper as a viable solution to reduce the complexity of the GNC algorithms for spacecraft formation flying. The main idea is that it is possible to drive the onboard actuators directly through the comparison of actual captured frames with reference images without the need to reconstruct the relative pose of the observed spacecraft. The projections of the visual features of the observed objects to the image plane are therefore used to close the feedback and the GNC loops.

In the paper, analytical developments demonstrate the stability of the proposed distributed visual servoing strategy, by also taking into account the orbital and attitude dynamics of the spacecraft. The presented approach is based on an optimal control framework for the minimization of the actuation efforts, which enables the generation of specific controllers addressing different tasks and issues in a variety of maneuvers, e.g., formation acquisition, formation reconfiguration or formation keeping maneuvers. The viability of the proposed control strategy, as well as the robustness against the errors in the actuation and sensing, is assessed through numerical simulations of a realistic scenario of small-sat formation flying.

In the following section (Section 2), a description of the particular formation flying scenario is provided together with the definition of the needed reference frames and the equations of motion that map the dynamics of the system. Section 3 defines the camera model and the observables that are used as input for designing the visual servoing controller. An ad-hoc optimized control is then developed in section 4 to address the formation acquisition and formation keeping tasks. Sections 5 assesses the performances and the robustness of such a controller against the more critical orbit 


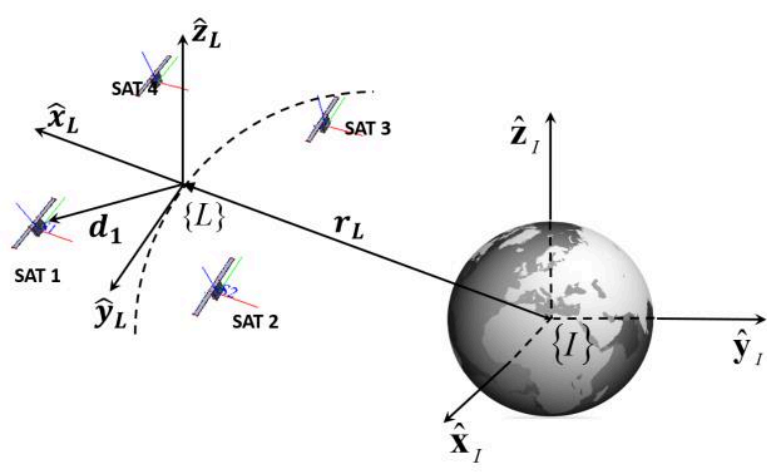

Fig. 1 Earth Centered Inertial and Local Vertical Local Horizontal reference frames for the 4 spacecraft formation flying scenario

perturbations and errors through numerical simulations. Finally, Section 6 summarizes the final findings and remarks as well as outlines possible developments and future investigations on this topic by the authors.

\section{SPACECRAFT FORMATION FLYING SCENARIO}

A scenario with $N=4$ spacecraft orbiting in formation as represented in Fig. 1 is considered as a baseline mission that is used for developing and specifying the algorithms.

Absolute positions and velocities of such spacecraft ( ${ }^{I} \boldsymbol{r}_{i}$ and ${ }^{I} \boldsymbol{v}_{i}$, with $i=1 \ldots N$ ) can be defined in an Earth Centered Inertial (ECI) reference frame that is denoted as $\{I\}$. Under the assumption that the inter-distances among the spacecraft, $d_{i j}=\left|\boldsymbol{r}_{i}-\boldsymbol{r}_{j}\right|$, are reasonably small if compared to any of the distances of spacecraft from the Earth's center $\left(\max \left(d_{i j}\right) \ll \min \left(r_{i}\right)\right.$, with $\left.i, j=1 \ldots N\right)$, it is possible to define a Local Vertical Local Horizontal (LVLH) reference frame, denoted as $\{L\}$, whose origin is initially centered to the center of the formation, as per:

$$
{ }^{\boldsymbol{I}} \boldsymbol{r}_{L}=\frac{\sum_{i=1}^{N}{ }^{I} \boldsymbol{r}_{i}}{N}
$$

with the unit vector ${ }^{I} \widehat{\boldsymbol{x}}_{L}$ aligned along the radial direction, ${ }^{I} \widehat{\boldsymbol{z}}_{L}$ along the orbit normal and ${ }^{I} \widehat{\boldsymbol{y}}_{L}$ along the in-track direction. Thus, the relative position of each spacecraft reads as:

$$
{ }^{L} \boldsymbol{d}_{i}={ }^{L} \boldsymbol{R}_{I}\left({ }^{I} \boldsymbol{r}_{i}-{ }^{I} \boldsymbol{r}_{L}\right)
$$

where ${ }^{L} \boldsymbol{R}_{I}$ is the cosine direction matrix between the $\{I\}$ and $\{L\}$ coordinate frame, defined as in [9].

The relative velocity of the $i$-th spacecraft $\left({ }^{L} \dot{\boldsymbol{d}}_{i}\right)$, calculated with respect to the rotating coordinate frame $\{L\}$, reads then as:

$$
{ }^{L} \dot{\boldsymbol{d}}_{i}={ }^{L} \boldsymbol{R}_{I}\left({ }^{I} \boldsymbol{v}_{i}-{ }^{I} \boldsymbol{v}_{L}\right)-{ }^{L} \boldsymbol{n}_{L} \times{ }^{L} \boldsymbol{d}_{i}
$$

where ${ }^{L} \boldsymbol{n}_{L}=n_{L}{ }^{L} \hat{\boldsymbol{z}}_{L}$, with $n_{L}=\sqrt{\mu_{E} / a_{L}^{3}}$ defined as the mean motion of the reference orbit, $\mu_{E}=398600 \mathrm{~km} / \mathrm{s}^{3}$ Earth's planetary constant and $a_{L}$ the semi-major axis of reference orbit.

Under the hypothesis that the reference orbit is circular or nearly-circular, a linearized set of equations of motion that also takes the main gravitational effects into account, has been found in [10] and it can be written as follows:

$$
{ }^{L} \ddot{\boldsymbol{d}}_{i}=\boldsymbol{A}_{1}{ }^{L} \boldsymbol{d}_{i}+\boldsymbol{A}_{2}{ }^{L} \dot{\boldsymbol{d}}_{i}+\boldsymbol{B}_{i}{ }^{L} \boldsymbol{t}_{i}
$$

where ${ }^{L} \boldsymbol{t}_{i}$ is the thrust applied to each $i$-th spacecraft defined in the LVLH frame, and $\boldsymbol{A}_{1}, \boldsymbol{A}_{2}$ and $\boldsymbol{B}_{i}$ read as:

$$
\begin{aligned}
\boldsymbol{A}_{1} & =\left[\begin{array}{ccc}
3 n_{L}^{2} & 0 & 0 \\
0 & 0 & 0 \\
0 & 0 & -n_{L}^{2}
\end{array}\right] \\
\boldsymbol{A}_{2} & =\left[\begin{array}{ccc}
0 & 2 n_{L} & 0 \\
-2 n_{L} & 0 & 0 \\
0 & 0 & 0
\end{array}\right] \\
\boldsymbol{B}_{i} & =\frac{1}{m_{i}}\left[\begin{array}{lll}
1 & 0 & 0 \\
0 & 1 & 0 \\
0 & 0 & 1
\end{array}\right]
\end{aligned}
$$

where $m_{i}$ is the mass of the spacecraft. It is worth noting that in Eq. (4) the thrust ${ }^{L} \boldsymbol{t}_{i}$ has its components defined with respect to the LVLH reference frame. However, thrusts are usually generated by thrusters attached to the body of the spacecraft. A new set of reference frames, defined as $\left\{S_{i}\right\}$, with $i=1,2, \ldots N$, can be defined in order to represent the actual attitude of each member of the formation. Such references are moving rigidly together with the satellites, as represented in Fig. 1.

If ${ }^{i} \boldsymbol{R}_{I}$ is the rotation matrix that defines the attitude of each spacecraft with respect to the inertial reference frame, the rotation matrix that transforms the thrusts from the body reference frames to the LVLH frame can be calculated as follows:

$$
{ }^{L} \boldsymbol{R}_{i}={ }^{L} \boldsymbol{R}_{I}{ }^{I} \boldsymbol{R}_{i}={ }^{L} \boldsymbol{R}_{I}{ }^{i} \boldsymbol{R}_{I}^{T}
$$

where the superscript $\mathrm{T}$ represents the transpose of the matrix. The rotation matrix ${ }^{i} \boldsymbol{R}_{I}$ can be defined in terms of quaternions $Q_{i}=\left[\begin{array}{ll}\boldsymbol{q}_{i} & q_{i}\end{array}\right]^{T}$ as follows ([11], pp.318-320):

$$
{ }^{i} \boldsymbol{R}_{I}=\left[\left(q_{i}^{2}-\boldsymbol{q}_{i}^{T} \boldsymbol{q}_{i}\right) \boldsymbol{E}+2 \boldsymbol{q}_{i} \boldsymbol{q}_{i}^{T}-2 q_{i} \widetilde{\boldsymbol{q}}_{i}\right]
$$

where $\boldsymbol{E}$ is the identity matrix and $\widetilde{\boldsymbol{q}}_{i}$ is the skew-symmetric matrix of the vector part of the quaternion. The kinematic equations concerning the spacecraft attitude are expressed using quaternions form:

$$
\dot{Q}_{\iota}=\frac{1}{2} \boldsymbol{\Omega}\left(\boldsymbol{\omega}_{i}\right) Q_{i}=\frac{1}{2}\left[\begin{array}{cc}
{ }^{i} \widetilde{\boldsymbol{\omega}_{\boldsymbol{\iota}}} & { }^{\boldsymbol{i}} \boldsymbol{\omega}_{i} \\
-{ }^{\boldsymbol{i}} \boldsymbol{\omega}_{i}^{T} & 0
\end{array}\right]\left[\begin{array}{l}
\boldsymbol{q}_{i} \\
q_{i}
\end{array}\right]
$$

where $\boldsymbol{\omega}_{i}$ is the angular velocity of the spacecraft.

The attitude dynamics of the spacecraft is modeled under the assumption that the spacecraft behave as rigid bodies subjected to environmental and control torques. The equations representing the attitude dynamics can be then written as follows:

$$
{ }^{i} \dot{\boldsymbol{\omega}}_{i}={ }^{i} \boldsymbol{J}_{i}^{-1}\left(-{ }^{i} \widetilde{\boldsymbol{\omega}}_{\imath}{ }^{i} \boldsymbol{J}_{i}{ }^{i} \boldsymbol{\omega}_{i}+{ }^{i} \boldsymbol{\tau}_{e}+{ }^{i} \boldsymbol{\tau}_{i}\right)
$$

where ${ }^{i} \boldsymbol{J}_{i}$ is the moment of inertia matrix of the spacecraft with respect to and expressed in the body frame, ${ }^{i} \boldsymbol{\tau}_{i}$ is the control torque and ${ }^{i} \boldsymbol{\tau}_{e}$ is the disturbing external torque applied to the satellite. 


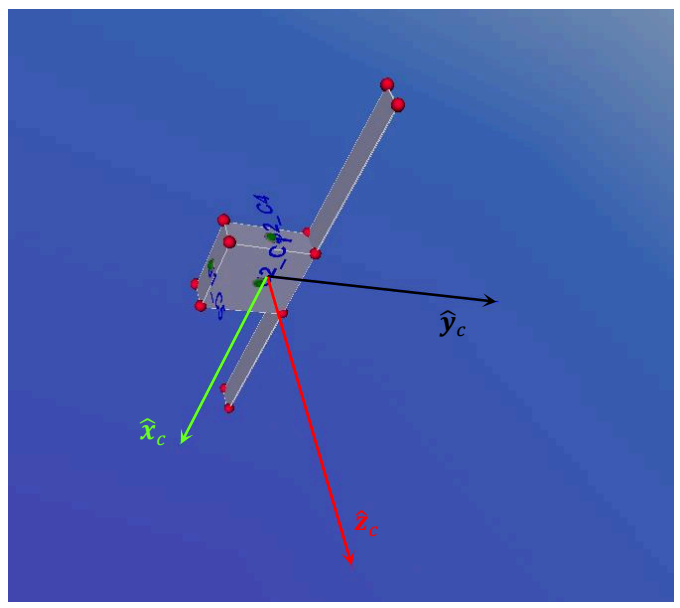

Fig. 2 Camera configuration within the body frame of a spacecraft of the formation

In this simplified model, each satellite is assumed being equipped with actuators to have full control of the orbit and attitude dynamics, e.g. thrusters and reaction wheels, respectively.

The system composed by Eqs. (4) and (11) represents the core of the equations of motion that characterizes each object in the model scenario, that can be rewritten as follows:

$$
\boldsymbol{F}_{c, i}+\boldsymbol{F}_{e, i}=\boldsymbol{I}_{i} \ddot{\boldsymbol{x}}_{i}+\boldsymbol{C}_{i}
$$

where $\ddot{\boldsymbol{x}}_{i}=\left[\begin{array}{ll}\ddot{\boldsymbol{d}}_{i}^{\mathrm{T}} & \dot{\boldsymbol{\omega}}_{i}^{\mathrm{T}}\end{array}\right]^{\mathrm{T}} \in \Re^{6}$ contains the absolute linear and angular accelerations of the $i$-th spacecraft, $\boldsymbol{I}_{i} \in \mathfrak{R}^{6 \times 6}$ is the mass matrix containing the mass and moments of the inertia of the spacecraft, $\boldsymbol{C}_{i} \in \mathfrak{R}^{6}$ contains the non-linear velocity/displacement-dependent terms, $\boldsymbol{F}_{c, i} \in \mathfrak{R}^{6}$ contains the force and moment exerted by the satellite actuators, and $\boldsymbol{F}_{e, i} \in \mathfrak{R}^{6}$ contains the external/disturbing forces and torques applied to the chaser satellite.

\section{Camera Model}

Each spacecraft is equipped with a system of cameras, as represented in Fig. 2, where the spacecraft body reference frame and one out of six camera frames are also illustrated. The latest has the ${ }^{i} \widehat{\mathbf{z}}_{c}$ axis aligned along the outward direction of the optical axis of the camera and the other two axes ( ${ }^{i} \widehat{\boldsymbol{x}}_{c \gamma}$ and ${ }^{i} \widehat{\boldsymbol{y}}_{c \gamma}$ ) laying on the outer panel surface of the panel. The same logic is repeated for defining each of the five other camera frames $\left\{C_{\gamma}\right\}$ of the satellite. Figure YY shows the ideal projection of a point $p$ on the image plane when it falls within the field of view of the camera. The relative position vector between the camera center $c$ and the point $p$, seen from the camera perspective, ${ }^{c} \boldsymbol{d}_{p c}=$ $\left[\begin{array}{lll}{ }^{c} x_{p c} & { }^{c} y_{p c} & { }^{c} z_{p c}\end{array}\right]^{T}$, can be computed as follows:

$$
{ }^{c} \boldsymbol{d}_{p c}={ }^{c} \boldsymbol{R}_{i}\left({ }^{i} \boldsymbol{R}_{I}{ }^{I} \boldsymbol{r}_{p}-{ }^{i} \boldsymbol{r}_{c}\right)
$$

where ${ }^{c} \boldsymbol{R}_{i}$ is the rotation matrix that rotates the components of a vector from the $i$-th satellite body frame to the camera frame. The projection of such a vector in the image plane will localize the position of the visual feature of such point $\boldsymbol{f}_{p} \in$ $\mathfrak{R}^{2}$ defined as:

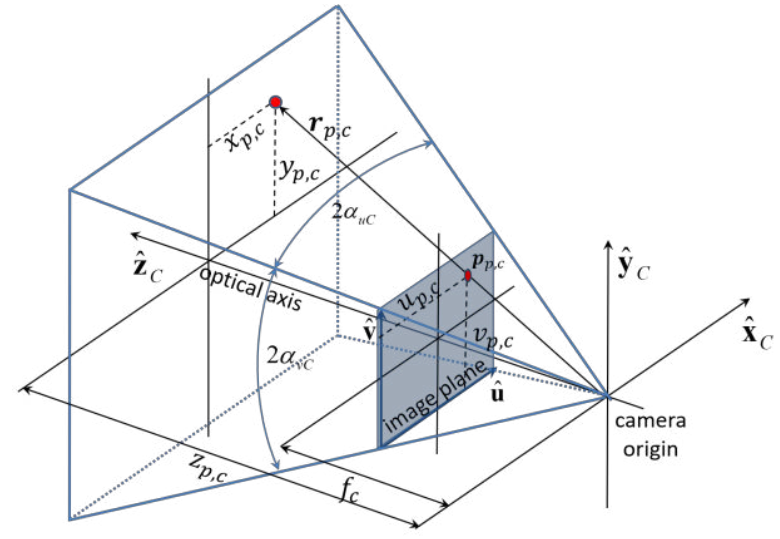

Fig. 3 Camera reference frame, image plane and image formation

$$
\boldsymbol{f}_{p}=\left[u_{p c}, v_{p c}\right]^{\mathrm{T}} \in \mathfrak{R}^{2}
$$

By assuming a pin-hole model of the camera, the two components $u_{p c}$ and $v_{p c}$ can be calculated as:

$$
\begin{aligned}
& u_{p c}=\frac{f_{c}}{\rho_{u c}} \frac{{ }^{c} x_{p c}}{{ }^{c} Z_{p c}}+u_{0 c} \\
& v_{p c}=\frac{f_{C}}{\rho_{u c}} \frac{{ }^{c} y_{p c}}{{ }^{c} Z_{p c}}+v_{0 c}
\end{aligned}
$$

where $f_{c}$ is the focal length of the camera, $\rho_{u c}$ and $\rho_{v c}$ are the width and the height of each pixel and, $u_{0 c}$ and $v_{0 c}$ define the center of the image plane, expressed in pixels.

This study considers that the features extraction from the observed object that falls within the field of view of the camera is made by computer vision algorithms [8] that is able to detect a certain number $\eta$ of visual features that can be organized in a matrix, as follows:

$$
\boldsymbol{s}=\left[\boldsymbol{f}_{1}, \boldsymbol{f}_{2}, \ldots, \boldsymbol{f}_{\eta}\right]^{\mathrm{T}} \in \mathfrak{R}^{2 \eta}
$$

\section{Optimal Visual Servoing Controller}

A distributed image-based visual servoing strategy is applied to the spacecraft in order to perform formation acquisition and keeping maneuvers. Such kind of control directly tracks desired trajectories of the visual features in the image plane, $s^{*}(t)$, without the need of estimating relative positions of the observed objects with filters or other estimation techniques. The result is a set of required linear and angular accelerations that can be directly applied to each of the spacecraft of the formation.

In order to reduce the energy and fuel required for performing the visual servoing task, the proposed optimal controller is designed to minimize the control action to each of the satellites, by taking into account the following function cost:

$$
\Omega_{i}=\boldsymbol{F}_{c, i}{ }^{T} \mathbf{W}_{i} \boldsymbol{F}_{c, i}
$$

where $\mathbf{W}_{i}$ is a time-dependent weight matrix. A set of $m$ constraints are used to define the visual servoing tasks that 
need to be executed during the maneuvers. These constraints can be generically defined as:

$$
\mathbf{A}_{i}(t) \ddot{\boldsymbol{x}}_{i}=\boldsymbol{b}_{i}(t)
$$

where $\mathbf{A}_{i}(t) \in \mathfrak{R}^{\mathrm{m} \times 6}$ and $\boldsymbol{b}_{i}(t) \in \mathfrak{R}^{\mathrm{m} \times 1}$. An advantage of this task formulation is that non-holonomic constraints can be treated in the same general way.

The control law that minimizes the cost function in Eq.(18), based on the dynamics model expressed in Eq. (12), while performing the tasks described in Eq.(19), is given by:

$$
\boldsymbol{F}_{c, i}=\boldsymbol{W}_{i}^{-1 / 2}\left(\mathbf{A}_{i} \boldsymbol{I}_{i}^{-1} \boldsymbol{W}_{i}^{-1 / 2}\right)^{+} \cdot\left(\boldsymbol{b}_{i}+\mathbf{A}_{i} \boldsymbol{I}_{i}^{-1} \boldsymbol{C}_{i}-\mathbf{A}_{i} \boldsymbol{I}_{i}^{-1} \boldsymbol{F}_{e, i}\right)
$$

where the symbol + denotes the pseudo-inverse of a general matrix.

Proof: By defining $\boldsymbol{z}=\boldsymbol{W}_{i}^{1 / 2} \boldsymbol{F}_{c, i}=\boldsymbol{W}_{i}^{1 / 2}\left(\boldsymbol{I}_{i} \ddot{\boldsymbol{x}}_{i}+\boldsymbol{C}_{i}-\boldsymbol{F}_{e, i}\right)$, it is possible to derive the linear and angular accelerations $\ddot{\boldsymbol{x}}_{i}=$ $\boldsymbol{I}_{i}^{-1}\left(\boldsymbol{W}_{i}^{-1 / 2} \boldsymbol{z}-\boldsymbol{C}_{c}+\boldsymbol{F}_{e, i}\right)$.

Taking into account the constraints defined in Eq.(19):

$$
\mathbf{A}_{i} \boldsymbol{I}_{i}^{-1} \boldsymbol{W}_{i}^{-1 / 2} \boldsymbol{z}=\boldsymbol{b}_{\boldsymbol{i}}+\mathbf{A}_{i} \boldsymbol{I}_{i}^{-1} \boldsymbol{C}_{i}-\mathbf{A}_{i} \boldsymbol{I}_{i}^{-1} \boldsymbol{F}_{e, i}
$$

The vector $\boldsymbol{z}$ which minimizes $\Omega=\boldsymbol{z}^{T} \boldsymbol{z}$ while fulfilling Eq. (21) is given by $\quad \boldsymbol{z}=\left(\mathbf{A}_{i} \boldsymbol{I}_{i}{ }^{-1} \boldsymbol{W}_{i}^{-1 / 2}\right)^{+}\left(\boldsymbol{b}_{i}+\mathbf{A}_{i} \boldsymbol{I}_{i}{ }^{-1} \boldsymbol{C}_{i^{-}}\right.$ $\left.\mathbf{A}_{i} \boldsymbol{I}_{i}{ }^{-1} \boldsymbol{F}_{e, i}\right)$, and as the force and moment exerted by the satellite actuators is given by $\boldsymbol{F}_{c, i}=\boldsymbol{W}_{\boldsymbol{i}}^{-1 / 2} \boldsymbol{z}$, so that Eq.(20) is consequently demonstrated.

The constraints in Eq.(19) are obtained by considering the visual servoing tasks. The goal of the image-based visual servoing controller is to track the desired trajectories of the selected $\eta$ visual features in the image plane so that the following equation is satisfied [12]:

$$
\left(\ddot{\boldsymbol{s}}^{*}-\ddot{\boldsymbol{s}}\right)+\boldsymbol{K}_{d}\left(\dot{\boldsymbol{s}}^{*}-\dot{\boldsymbol{s}}\right)+\boldsymbol{K}_{p}\left(\boldsymbol{s}^{*}-\boldsymbol{s}\right)=0
$$

where $\ddot{\boldsymbol{s}}^{*}, \dot{\boldsymbol{s}}^{*}$ and $\mathbf{s}^{*}$ are the desired accelerations, velocities and positions of the visual features in the image plane, respectively. $\boldsymbol{K}_{p}$ and $\boldsymbol{K}_{d}$ are proportional and derivative gain matrices, respectively. Thus, Eq. (22) can be expressed in terms of image errors as follows:

$$
\ddot{\boldsymbol{s}}^{*}+\boldsymbol{K}_{d} \dot{\boldsymbol{e}}_{s}+\boldsymbol{K}_{p} \boldsymbol{e}_{s}=\ddot{\boldsymbol{s}}_{r}
$$

where $\boldsymbol{e}_{\boldsymbol{s}}$ and $\dot{\boldsymbol{e}}_{\boldsymbol{s}}$ are the image error and time derivative of the image error, respectively.

The relationship between the velocities of the $\eta$ extracted visual features in the camera image plane $\left(\dot{\boldsymbol{s}}_{r} \in \mathfrak{R}^{2 \eta}\right)$ and the angular and linear velocities of the camera $\left(\dot{\boldsymbol{x}}_{c} \in \mathfrak{R}^{6}\right)$ is described by the following equation:

$$
\dot{\boldsymbol{s}}_{r}=\mathbf{L}_{s} \dot{\boldsymbol{x}}_{\mathrm{c}}
$$

where $\mathbf{L}_{s} \in \mathfrak{R}^{2 \eta \times 6}$ is the interaction matrix that relates the velocities of image feature points to the camera velocity vector [8]. This interaction matrix gathers all the interaction matrices related to each image feature in the image, as follows:

$$
\mathbf{L}_{s}=\left[\begin{array}{llll}
\mathbf{L}_{s 1}^{T} & \mathbf{L}_{s 2}^{T} & \ldots & \mathbf{L}_{s \eta}^{T}
\end{array}\right]^{T}
$$

where each $\mathbf{L}_{s k}$ is the $2 \times 6$ interaction matrix of each image feature $\mathrm{k}=1,2 \ldots, \eta$.
The image acceleration or second derivative of sr is obtained by differentiating Eq.(24) with respect to time, as follows:

$$
\ddot{\boldsymbol{s}}_{r}=\mathbf{L}_{s} \ddot{\boldsymbol{x}}_{\mathrm{c}}+\dot{\mathbf{L}}_{s} \dot{\boldsymbol{x}}_{\mathrm{c}}
$$

Therefore, the visual servoing task is defined by the following relationships:

$$
\begin{gathered}
\mathbf{A}_{i}=\mathbf{L}_{s} \\
\boldsymbol{b}_{\boldsymbol{i}}=\ddot{\boldsymbol{s}}_{r}-\dot{\mathbf{L}}_{s} \dot{\boldsymbol{x}}_{\mathrm{c}}
\end{gathered}
$$

Thus, the final control law is obtained replacing Eqs.(27) and (28) into Eq.(20):

$$
\begin{gathered}
\boldsymbol{F}_{c, i}=\boldsymbol{W}_{i}^{-1 / 2}\left(\mathbf{L}_{s} \boldsymbol{I}_{i}^{-1} \boldsymbol{W}_{i}^{-1 / 2}\right)^{+} \\
\left(\ddot{\boldsymbol{s}}_{r}-\dot{\mathbf{L}}_{s} \dot{\boldsymbol{x}}_{\mathrm{c}}+\mathbf{L}_{s} \boldsymbol{I}_{i}^{-1} \boldsymbol{C}_{i^{-}} \mathbf{L}_{s} \boldsymbol{I}_{i}^{-1} \boldsymbol{F}_{e, i}\right)
\end{gathered}
$$

It is worth noting that the choice of $\boldsymbol{W}_{i}$ plays an important role in the controller because determines how the control action is distributed by the actuators. Further, some values of this matrix can simplify the product $\left(\mathbf{L}_{s} \boldsymbol{I}_{i}{ }^{-1} \boldsymbol{W}_{i}^{-1 / 2}\right)^{+}$and consequently, the control law. Indeed, a wide range of, visual servoing control laws can be obtained from the choice of different values of $\boldsymbol{W}_{i}$.

The first control is obtained by imposing $\boldsymbol{W}_{i}=\boldsymbol{I}_{i}^{-2}$ in Eq.(29):

$$
\boldsymbol{F}_{c, i}=\boldsymbol{I}_{i} \boldsymbol{L}_{s}{ }^{+} \cdot\left(\ddot{\boldsymbol{s}}_{r}-\dot{\mathbf{L}}_{s} \dot{\boldsymbol{x}}_{\mathrm{c}}+\mathbf{L}_{s} \boldsymbol{I}_{i}{ }^{-1} \boldsymbol{C}_{c^{-}} \mathbf{L}_{s} \boldsymbol{I}_{i}^{-1} \boldsymbol{F}_{e, i}\right)
$$

Additionally, by setting $\boldsymbol{W}_{\boldsymbol{i}}=\boldsymbol{I}_{c}{ }^{-1}$ we can obtain:

$$
\begin{gathered}
\boldsymbol{F}_{c, i}=\boldsymbol{I}_{i}^{1 / 2}\left(\mathbf{L}_{\mathrm{s}} \boldsymbol{I}_{i}^{-1} \boldsymbol{I}_{i}^{1 / 2}\right)^{+} \\
\left(\ddot{\boldsymbol{s}}_{r}-\dot{\mathbf{L}}_{\mathrm{s}} \dot{\boldsymbol{x}}_{\mathrm{c}}+\boldsymbol{L}_{s} \boldsymbol{I}_{i}^{-1} \boldsymbol{C}_{c}-\mathbf{L}_{\mathrm{s}} \boldsymbol{I}_{i}^{-1} \boldsymbol{F}_{e, i}\right)
\end{gathered}
$$

Finally, in the results section, these controllers will be compared with the simplest case, i.e., the weighting matrix equal to the identity matrix

\section{NUMERICAL SimUlationS}

A simulation setup has been developed in order to assess the suitability of the proposed visual servoing strategies in a close formation flying scenario. A formation of $N=4$ spacecraft is taken as a test case scenario, with an initial configuration shown in Fig. 4. The initial positions and

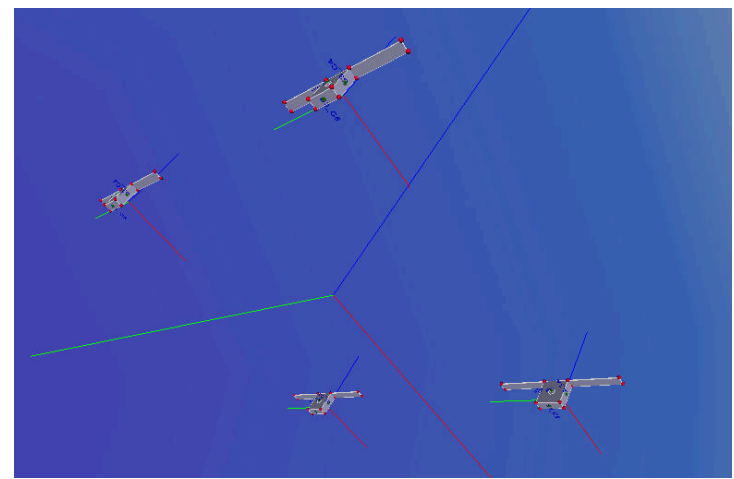

Fig. 4 Initial configuration of the spacecraft formation scenario 


\begin{tabular}{c|cc|c} 
& \multicolumn{2}{|c|}{ Position $(\mathrm{m})$} & \multicolumn{2}{c}{ Velocity $(\mathrm{m} / \mathrm{s})$} \\
\hline \hline Sat 1 & {$\left[\begin{array}{lll}200.0 & 200.0 & 0.0\end{array}\right]^{T}$} & {$\left[\begin{array}{llll}0.0 & 0.0 & 0.0\end{array}\right]^{T}$} \\
\hline Sat 2 & {$\left[\begin{array}{lll}-200.0 & 200.0 & 0.0\end{array}\right]^{T}$} & {$\left[\begin{array}{lll}0.0 & 0.0 & 0.0\end{array}\right]^{T}$} \\
\hline Sat 3 & {$\left[\begin{array}{lll}-200.0 & -200.0 & 0.0\end{array}\right]^{T}$} & {$\left[\begin{array}{lll}0.0 & 0.0 & 0.0\end{array}\right]^{T}$} \\
\hline Sat 4 & {$\left[\begin{array}{lll}200.0 & -200.0 & 0.0\end{array}\right]^{T}$} & {$\left[\begin{array}{llll}0.0 & 0.0 & 0.0\end{array}\right]^{T}$}
\end{tabular}

Table 1 Initial spacecraft configuration of the formation w.r.t. the LVLH reference frame

\begin{tabular}{c|c} 
Mass (kg) & $\begin{array}{c}\text { Moments of } \\
\text { Inertia }\left(\mathrm{kg} \cdot \mathrm{m}^{2}\right)\end{array}$ \\
\hline \hline \multirow{2}{*}{50} & {$\left[\begin{array}{ccc}57.6 & 0.0 & 0.0 \\
0.0 & 64.8 & 0.0 \\
0.0 & 0.0 & 72.0\end{array}\right]$}
\end{tabular}

Table 2 Mass properties of the spacecraft of the formation

velocities are reported in Table 1, while the initial attitudes are taken in such a way that the satellites' body frames are initially aligned as the LVLH frame.Each of the spacecraft has the mass properties as reported in Table 2 .

For the specific investigation in this paper, formation keeping and formation reconfiguration maneuvers have been taken as a test case maneuvers in order to evaluate the performances of such techniques. It is also worth to note that a restrictive assumption was necessary for performing the simulations. Each of the satellites can detect specific visual features (red points in Fig. 4) of only one of the spacecraft belonging to the formation. Namely, Sat 1 is able to see the visual features of Sat 2, Sat 2 has the camera oriented towards Sat 3, Sat 3 is able to detect features of Sat 4, and so will do Sat 4 with Sat 1 . The authors will perform further investigations in order to remove and make more generic the adopted approach.

\section{A. Formation keeping maneuver}

The aim of this maneuver to maintain the initial relative configuration of the formation even though the spacecraft are subjected to environmental actions. In order, to perform such maneuvers, the camera of each spacecraft initially captures the visual features of the observed spacecraft, as shown in Fig. 5, and the algorithm will try to maintain the relative geometry among these features for all the duration of the maneuver, compensating the effects of the relative orbital motion and perturbations. The visual servoing controller adopts $\mathbf{K}_{p}=$ $0.001 E$ and $\mathbf{K}_{d}=0.05 E$ as proportional and derivative gain matrices, respectively. Further, the value of the weighting function $\boldsymbol{W}$ considered in this simulation is set as $\boldsymbol{I}_{c}^{-1}$
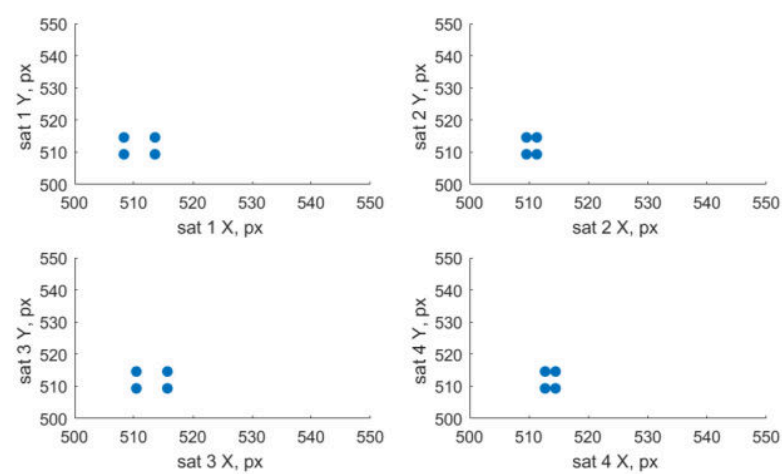

Fig. 5 Position of the extracted features in the image plane during the initial phase of the formation keeping maneuver

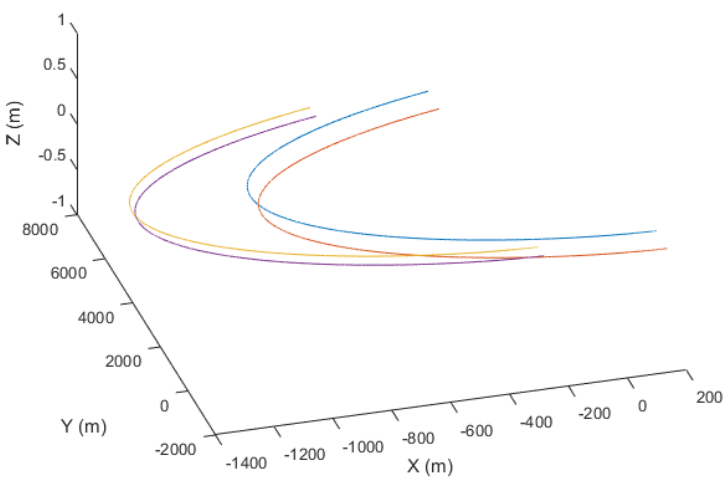

Fig. 6 Trajectories of the spacecraft w.r.t the LVLH coordinate frame during the formation keeping maneuver.
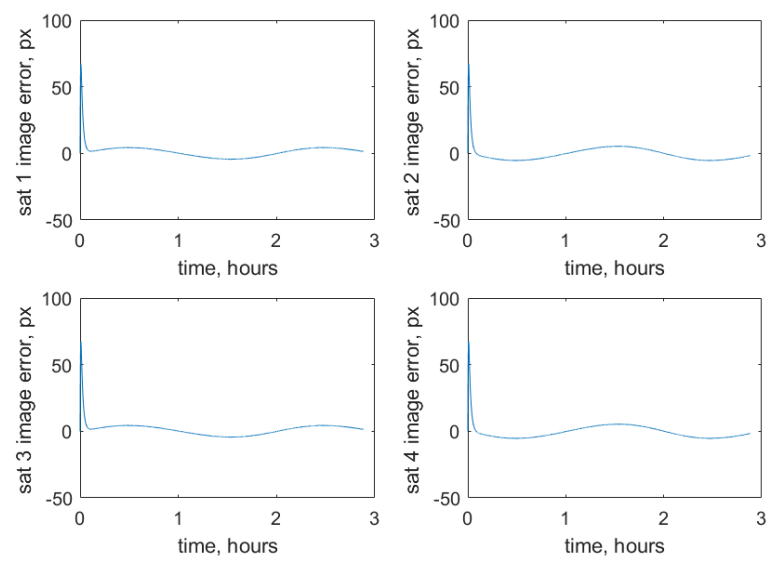

Fig. 7 Image error during the formation keeping maneuver

$\left(\boldsymbol{W}=\boldsymbol{I}_{c}{ }^{-1}\right)$. The 3D trajectory of the spacecraft during the formation keeping maneuver is represented in Fig. 6.

During the maneuver, the mean image error remains low once the initial perturbation is compensated, as shown in Fig. 7. As a consequence, the controller tries to keep the relative distance among the platforms constant. Fig. 8 shows the relative distance between the spacecraft. It is worth noting that such distances are indeed maintained around the desired 

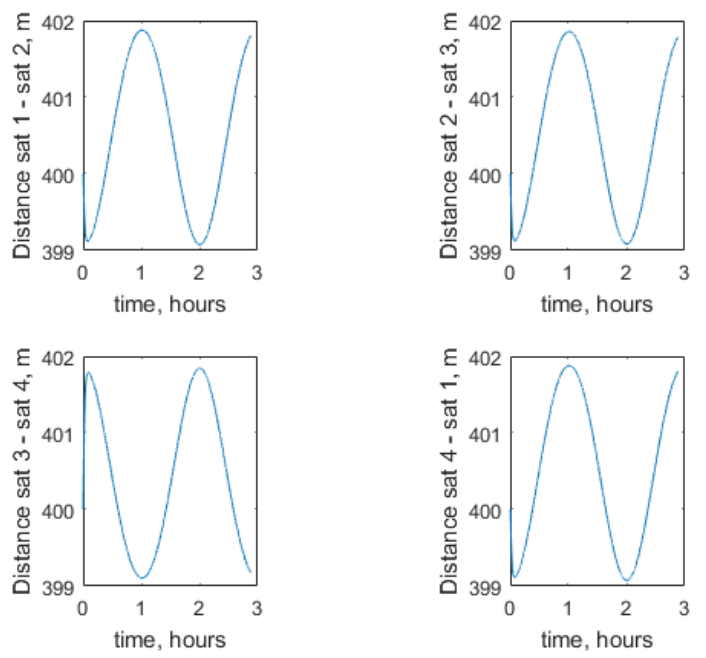

Fig. 8 Relative distance between the spacecraft during the formation keeping maneuver
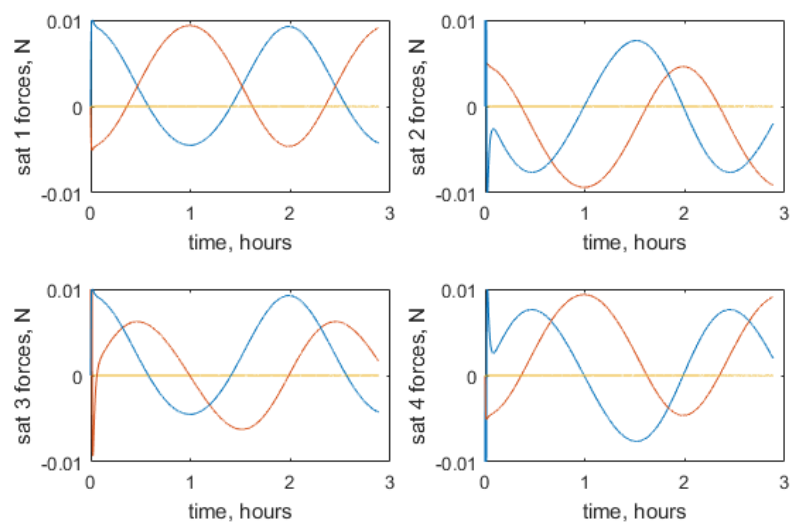

Fig. 9 Forces during the formation keeping maneuver

distance (400 m), with a slight modulation due to the changes in gravity gradient when the spacecraft switch positions each other with respect to the LVLH frame. This effect also affects the modulation of the thrusts applied to the spacecraft, represented in Fig. 9, that are indeed synchronized with the overall spinning motion of the formation w.r.t. the LVLH coordinate frame. This configuration is maintained for 3 hours. Three different cases have been analyzed in order to assess the performance of using different weighting matrices $\boldsymbol{W}_{\boldsymbol{i}}$. Table 3 shows the mean image error and $\Delta v$ obtained during these simulations. It is worth to note that the best result

\begin{tabular}{c|c|c}
$\mathbf{W}$ & Mean image error $(\mathbf{P x})$ & $\Delta \boldsymbol{v}(\mathbf{m} / \mathbf{s})$ \\
\hline \hline $\boldsymbol{I}_{i}{ }^{-1}$ & 2.81 & 5.61 \\
$\boldsymbol{I}_{i}{ }^{-2}$ & 3.78 & 6.38 \\
$\boldsymbol{E}$ & 3.80 & 6.42
\end{tabular}

Table 3 Performance of the formation keeping maneuver for different values of $\mathrm{W}$
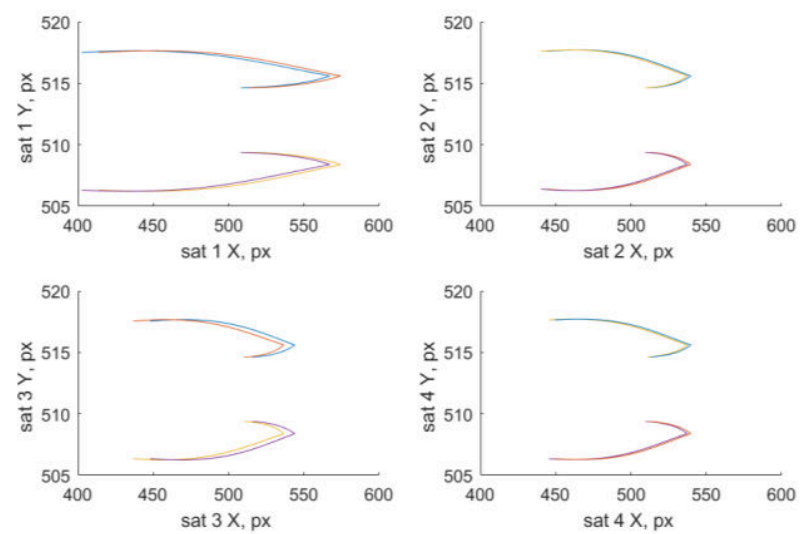

Fig. 10 Image trajectory during the formation reconfiguration maneuver

is obtained when $\boldsymbol{W}_{\boldsymbol{i}}=\boldsymbol{I}_{i}{ }^{-1}$, as both the mean image error and the $\Delta v$ are the lowest one.

\section{B. Formation reconfiguration maneuver}

The aim of the second maneuver is to perform a formation reconfiguration that will reduce the mutual distances of the spacecraft from the initial value of $400 \mathrm{~m}$ to a desired range of $180 \mathrm{~m}$. By using the initial configuration given in Table 1 , the cameras will shoot again the same initial configuration of the visual features as in Fig. 5. From this initial position of the spacecrafts the extracted image feature points are represented in Fig. 1. To perform this maneuver, the visual-servoing controllers need to track a desired trajectory that linearly spreads the visual features in the image plane up to the following desired values:

$\boldsymbol{s}_{d 1}=[(413,517),(402,517),(402,506),(413,506)]^{T} \mathrm{px} ;$ $\boldsymbol{s}_{d 2}=[(444,517),(440,517),(440,506),(444,506)]^{T} \mathrm{px}$; $\boldsymbol{s}_{d 3}=[(448,517),(437,517),(437,506),(448,506)]^{T} \mathrm{px} ;$ $\boldsymbol{s}_{d 4}=[(449,517),(445,517),(445,506),(449,506)]^{T} \mathrm{px}$.

The proportional and derivative gain matrices are $\mathbf{K}_{p}=$ $0.004 \boldsymbol{E}$ and $\mathbf{K}_{d}=0.01 \boldsymbol{E}$, respectively. The final image trajectories drawn in the image planes by the observed visual features of the spacecraft are shown in Fig. 10. The controllers compensate for the effects of the gravity gradient while they try to follow the desired trajectories in the image planes, tracing as a result evident arcs in the image planes. The maneuver is in any case successful, as shown in Fig. 11, where the relative distances between the spacecraft during the maneuver are represented. Fig. 12 shows the resulting

\begin{tabular}{c|c|c}
$\mathbf{W}$ & Mean image error $(\mathbf{P x})$ & $\Delta \boldsymbol{v}(\mathbf{m} / \mathbf{s})$ \\
\hline \hline $\boldsymbol{I}_{i}{ }^{-1}$ & 3.23 & 15.26 \\
$\boldsymbol{I}_{i}{ }^{-2}$ & 4.28 & 17.34 \\
$\boldsymbol{E}$ & 4.32 & 17.4
\end{tabular}

Table 4 Performance of the formation reconfiguration maneuver for different values of $\mathrm{W}$ 

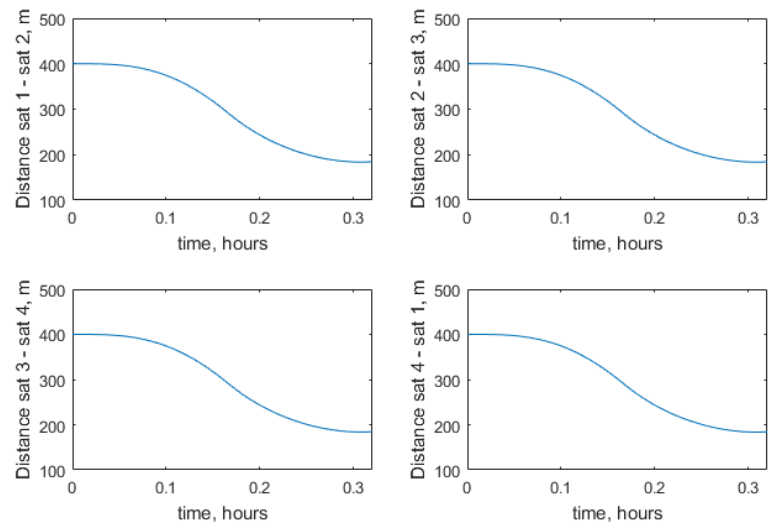

Fig. 11 Relative distance between the spacecrafts during the formation reconfiguration maneuver
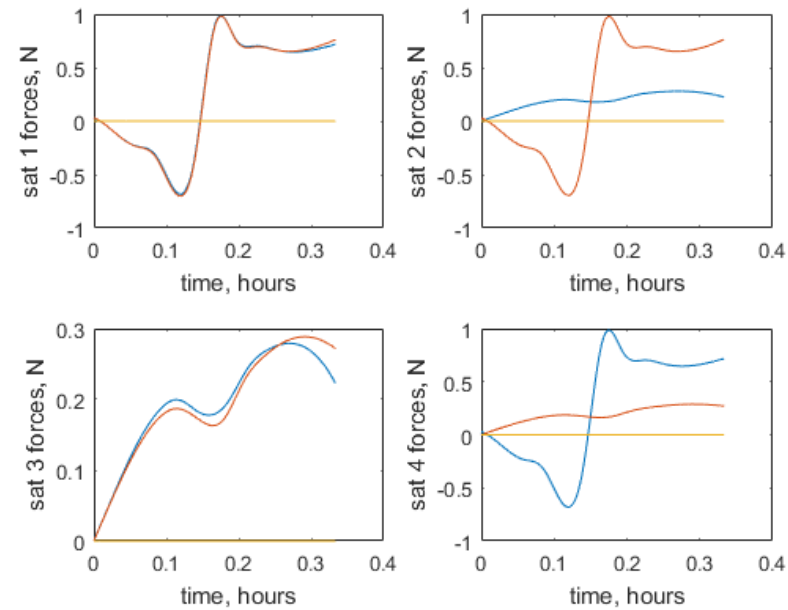

Fig. 12 Forces during the formation reconfiguration maneuver

behavior of the continous forces applied to the spacecraft for performing such kind of maneuvers.

Table 4 shows the performance of the visual servoing controller, measured against changes in the weighting matrix $\boldsymbol{W}_{\boldsymbol{i}}$. It is worth noting that the mean image errors are slightly increased and the $\Delta \boldsymbol{v}$-s are three times bigger, if compared with the values in Table 3 , but this is obviously a consequence of the different nature of the tasks that need to be performed in this maneuver. Hovewer, even in this case, the best results are obtained by setting $\boldsymbol{W}_{\boldsymbol{i}}=\boldsymbol{I}_{i}{ }^{-1}$.

In the previous cases, the four satellites were perfectly aligned in the same plane. In order to demonstrate the correct behavior of the presented approach when the formation includes out-of-plane satellites, a new experiment is presented in the next paragraphs. In this case, one of the satellites (sat 1) is $5 \mathrm{~m}$ out of the plane defined by the other three satellites. The proportional, derivative and weight matrix considered in this maneuver are $\mathbf{K}_{p}=0.004 \boldsymbol{E}, \mathbf{K}_{d}=$ $0.01 \boldsymbol{E}, \boldsymbol{W}_{\boldsymbol{i}}=\boldsymbol{I}_{i}^{-1}$ respectively.

Fig. 13 shows the image trajectories obtained with these new initial conditions. It is worth to notice that the image
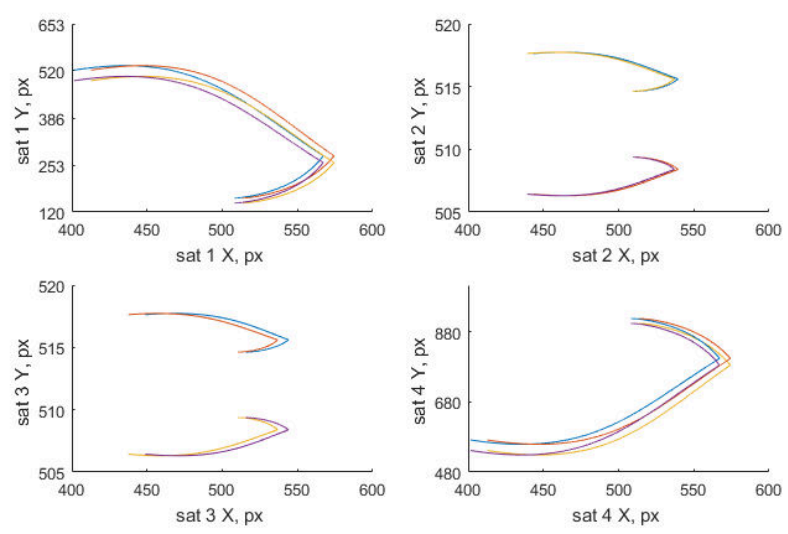

Fig. 13 Image trajectory during the formation reconfiguration maneuver (out-of-plane experiment)
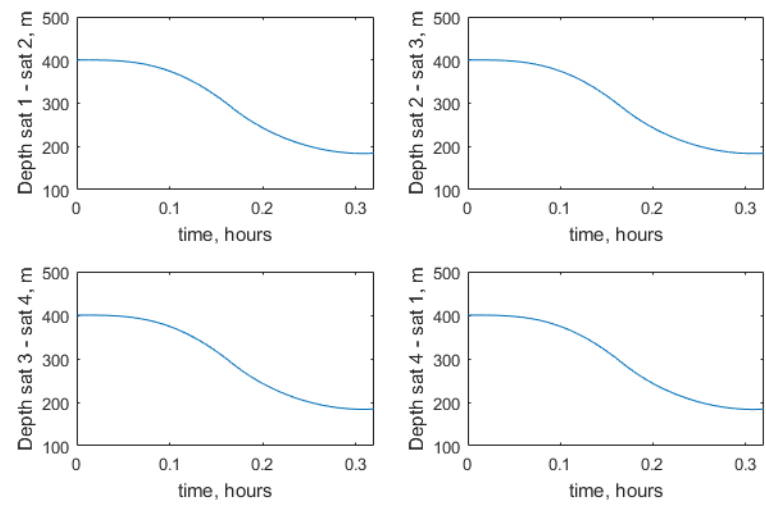

Fig. 14 Relative distance between the spacecrafts during the formation reconfiguration maneuver (outof-plane experiment)

trajectories for the Sat 1 and Sat 4 are different if compared with respect to Fig. 10: this is due to the initial deviation of Sat 1. However, the desired positions of the image features are in any case achieved, as shown in Fig. 13. Fig. 14 shows the relative distance among the satellites during the maneuver: the behavior of such distances is similar to the ones obtained in the previous case (see Fig. 11 for comparison). Finally, Fig. 15 shows the forces applied to the spacecrafts during this maneuver, where small forces appear in the $\mathrm{z}$ direction in order to compensate the out-of-plane deviation.

\section{Conclusion}

A computational efficient framework for generating optimal visual-servoing controllers to be applied on spacecraft formation was developed and presented in this paper. Such kind of method takes as input images from onboard cameras and directly uses them to command specific maneuvers. Analytical developments demonstrated the stability and optimality of the proposed distributed visual servoing strategy by taking into account the orbital and attitude dynamics of the spacecraft. Therefore, three different typologies of controllers have been generated and applied in 

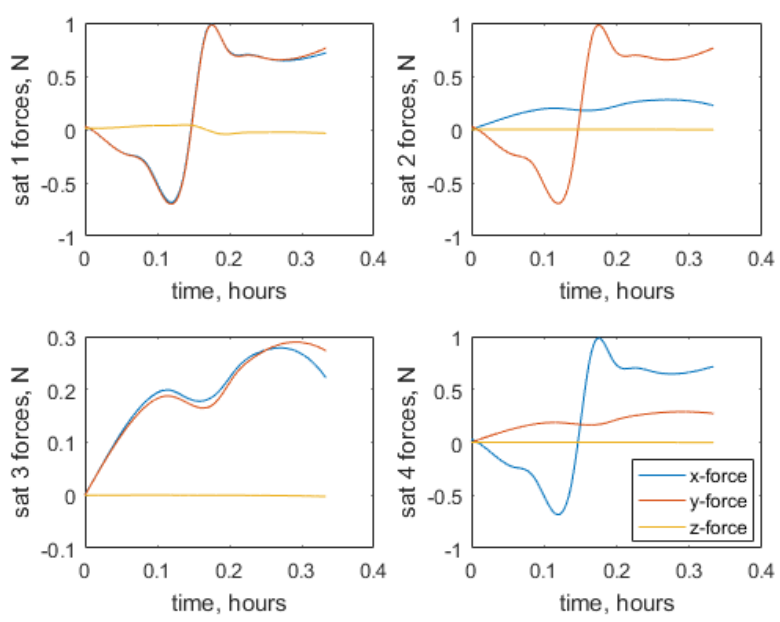

Fig. 15 Forces during the formation reconfiguration maneuver (out-of-plane experiment)

two different kinds of maneuvers, i.e. formation keeping and formation reconfiguration in a scenario that included four spacecraft. The viability of the proposed control strategy were assessed through numerical simulations, as well as performances are measured in terms of accuracy of the maneuvers and $\Delta \boldsymbol{v}$ needed to perform them. From the analysis of the results, it appeared evident that a control scheme that adopts a weight matrix $\boldsymbol{W}_{\boldsymbol{i}}=\boldsymbol{I}_{i}{ }^{-1}$ guaranties the best performances in both the maneuvers.

Further investigations of the authors will try to extend such an algorithm to more generic cases, including multiple observations of different spacecraft obtained from the same camera and generalizing the convergence criteria of such a distributed scheme by considering the topological properties of the formation.

\section{REFERENCES}

[1] G. B. Palmerini, "Relative navigation in autonomous spacecraft formations," 2016 IEEE Aerospace Conference, Big Sky, MT, 2016, pp. 1-10.

doi: 10.1109/AERO.2016.7500944

[2] S. D'Amico, J.-S. Ardaens, R. Larsson. "Spaceborne Autonomous Formation-Flying Experiment on the PRISMA Mission", Journal of Guidance, Control, and Dynamics, Vol. 35, No. 3 (2012), pp. 834-850. doi: $10.2514 / 1.55638$

[3] S. Segal, P. Gurfil and K. Shahid, "In-Orbit Tracking of Resident Space Objects: A Comparison of Monocular and Stereoscopic Vision," in IEEE Transactions on Aerospace and Electronic Systems, vol. 50, no. 1, pp. 676-688, January 2014

doi: 10.1109/TAES.2013.120006

[4] S. Sharma, J. Ventura, S. D’Amico. "Robust ModelBased Monocular Pose Initialization for Noncooperative Spacecraft Rendezvous", Journal of Spacecraft and Rockets, Vol. 55, No. 6 (2018), pp. 1414-1429. doi: 10.2514/1.A34124
[5] S. Llorente, A. Agenjo, C. Carrascosa, C. de Negueruela, A. Mestreau-Garreau, A. Cropp, A. Santovincenzo, PROBA-3: Precise formation flying demonstration mission, Acta Astronautica, Vol. 82, No 1, 2013, pp. 3846. doi:10.1016/j.actaastro.2012.05.029.

[6] R. Volpe, G. B. Palmerini, M. Sabatini, A passive camera based determination of a non-cooperative and unknown satellite's pose and shape, Acta Astronautica, Vol. 151, 2018, pp. 805-817 doi: 10.1016/j.actaastro.2018.06.061

[7] A. Poghosyan, A. Golkar, CubeSat evolution: Analyzing CubeSat capabilities for conducting science missions, Progress in Aerospace Sciences, Vol. 88, 2017, pp. 5983, doi:10.1016/j.paerosci.2016.11.002.

[8] F. Chaumette and S. Hutchinson, "Visual servo control. II. Advanced approaches," in IEEE Robotics \& Automation Magazine, vol. 14, no. 1, pp. 109-118, March 2007, doi: 10.1109/MRA.2007.339609

[9] J. Pomares, L. Felicetti, J. Pérez, M. R. Emami, Concurrent image-based visual servoing with adaptive zooming for non-cooperative rendezvous maneuvers, Advances in Space Research, Vol. 61, No. 3, 2018, pp. 862-878, doi: 10.1016/j.asr.2017.10.054

[10] W. Clohessy, R. Wiltshire, Terminal guidance system for satellite rendezvous, Journal of Aerospace Sciences Vol. 27, 1960, pp. 653-658, doi: 10.2514/8.8704

[11]Wie, B., 1998. Space Vehicle Dynamics and Control, AIAA Education Series

[12] J. Pomares, L. Felicetti, J. Perez and M. R. Emami, "Spacecraft visual servoing with adaptive zooming for non-cooperative rendezvous," 2018 IEEE Aerospace Conference, Big Sky, MT, 2018, pp. 1-8. doi: 10.1109/AERO.2018.8396472 


\section{BIOGRAPHY}

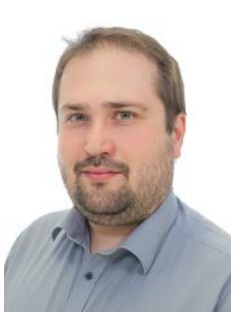

Leonard Felicetti is a Lecturer in Space Engineering in Cranfield University (UK). He obtained his Ph.D. and he was a Post-Doc Researcher in Sapienza - University of Rome (Italy). In 2015, he was Honorary Research Associate at University of Glasgow $(U K)$ and then, Associate Senior Lecturer in On-board Space Systems in

Luleå University of Technology (Sweden). He joined Cranfield University (UK) in 2019.Leonard's main research interests are on Spacecraft Formation Flying; Guidance, Navigation and Control; Spacecraft Orbital and Attitude Control; Space Robotics; Autonomous Distributed Space Systems; Spacecraft and Mission Design.

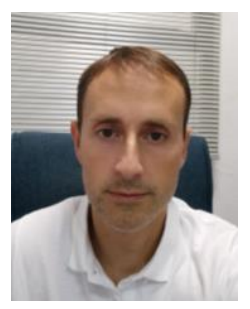

Jorge Pomares received the engineer and Ph.D. degrees in computer science from the University of Alicante, Spain in 1999 and 2004 respectively. He is full professor in engineering control and robotics at the Department of Physics, System Engineering and Signal Theory of the University of Alicante. His current research interests include robotics and control, especially visual servoing, space robotics, humanoids robots and advanced control of dynamic systmes. He was engaged in more than 20 advanced $R+D$ robotic projects, being main researcher on several of them. He is the author of a hundred publications in journals and conferences. 
2020-08-21

\section{Image-based visual servoing control for spacecraft formation flying}

Felicetti, Leonard

IEEE

Felicetti L, Pomares J. (2020) Image-based visual servoing control for spacecraft formation flying. In: 2020 IEEE Aerospace Conference, 7-14 March 2020, Big Sky, MT, USA

https://doi.org/10.1109/AERO47225.2020.9172494

Downloaded from Cranfield Library Services E-Repository 